\title{
Photonic processing subsystem based on visual cortex architecture
}

\author{
José A. Martín-Pereda ${ }^{1}$, Ana P. González-Marcos ${ }^{2}$ \\ E.T.S. Ingenieros de Telecomunicación. Universidad Politécnica de Madrid \\ Ciudad Universitaria. 28040 Madrid. Spain
}

\begin{abstract}
Optical signal processing in any living being is more complex than the one obtained in artificial systems. Cortex architecture, although only partly known, gives some useful ideas to be employed in communications. To analyze some of these structures is the objective of this paper. One of the main possibilities reported is handling signals in a parallel way. As it is shown, according to the signal characteristics each signal impinging onto a single input may be routed to a different output. At the same time, identical signals, coming to different inputs, may be routed to the same output without internal conflicts. This is due to the change of some of their characteristics in the way out when going through the intermediate levels. The simulation of this architecture is based on simple logic cells. The basis for the proposed architecture is the five layers of the mammalian retina and the first levels of the visual cortex.
\end{abstract}

Keywords: Photonic processing, visual cortex, parallel processing.

\section{INTRODUCTION}

One of the main characteristics of the visual system in almost any living being is the possibility to analyze complex images in a parallel way. This visual system is relatively quick, highly robust and effortless. Its basic characteristics are not found in existing computer-based systems that have been developed over the years for machinevision systems. These systems are not very efficient because the computational power required for processing and extracting features from a typical time-space varying visual scene is immense. As it is shown in ${ }^{1}$, if only one instruction per pixel is to be performed on a standard digital image containing $3 / 4$ million pixels that change at a rate of thirty frames per second, then a software-based vision algorithm implemented on a digital computer would be required to perform approximately 23 million instructions per second. Any realistic vision algorithm involves far more than a single instruction per pixel. This is in strong contrast to anyone experience where is able to perceive and provide a meaning to a complex time-varying scene in approximately $100 \mathrm{~ms}$ using a very slow information-processing element: the neuron. Response time to generate a response in any neuron is around 2 milliseconds. Moreover, many aspects of animal vision are achieved in only 20 to 50 transformation steps, far fewer than the millions of transformation operations required by a sequential algorithm implemented on a conventional digital computer.

To achieve above mentioned goals, living beings employ a computational architecture that is very different from that of a digital computer. Almost $60 \%$ of the sensory information received is projected through the visual pathway though a parallel processing. The visual information generated on the photoreceptors undergoes large processing an reorganization as it goes through the visual path to the visual cortex. Hence, the visual pathway can be seen as a large ensemble of parallel neuronal channel that transducer, transmit and process visual information. Each successive layer along this pathway performs a specific set of computational operations on incoming signals. Animal vision employs not only massively parallel but also hierarchical information-processing architecture. As information goes from the retina to deeper cortical regions, visual attributes contained in a scene become more and more abstract. This process culminates at certain cortical neurons that respond selectively to complex objects.

From these ideas, the study of animal vision provides a motivation and a general framework for designing a fast vision system and a method to process information, both in vision systems and in transmission systems. The main objective should be replicating some of the neuronal computational structures found in biological vision that are involved in processing, storing and interpreting the spatio-temporal visual information. In order to achieve this goal, this paper will be divided as follows. Section 2 is a brief overview of the living beings system from the neuron to the neuronal tissue of the visual pathway, including the retina and the primary visual cortex as well as some of their main

\footnotetext{
${ }^{1}$ jamp@tfo.upm.es

2 agonmar@tfo.upm.es
} 
characteristics for pattern recognition. In Section 3, we present a simple model to extract and interpret some straightforward properties of images. Section 4 will be devoted to apply this model to the transmission of parallel information through a communication channel.

\section{SOME BASIS OF THE MAMMALIAN VISUAL CORTEX}

This paper is not related with physiological aspects concerning the mammalian visual cortex. But because we considerer convenient to remember some of its main aspects we will present here some structural and functional details of this particular zone. Some of these aspects will be the basis for the present work. Moreover, because information transmission through different layers of the visual system is partly coded, some brief information will be given concerning
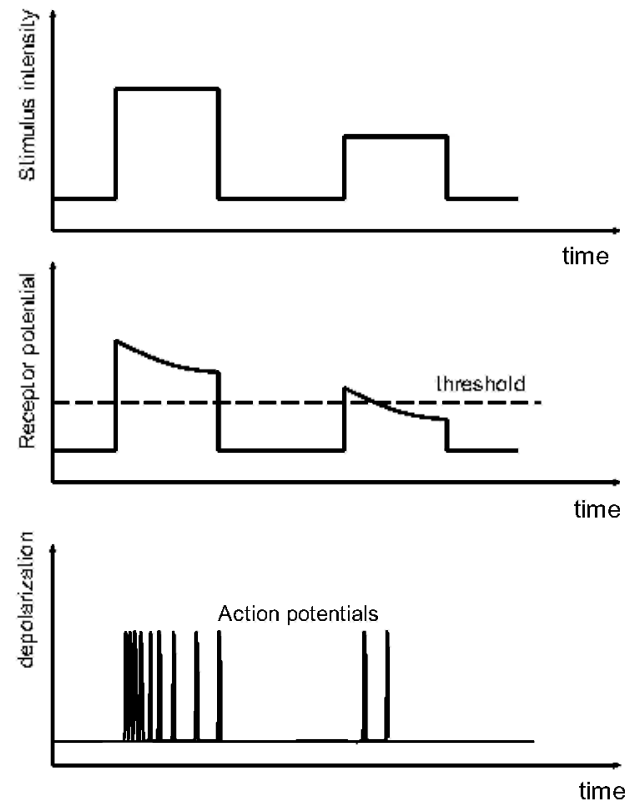

Fig. 1.- Relationships between stimulus intensity and frequency of action potentials some aspects of this transmission.

\subsection{Responses of the neurosensors}

Living bodies experience their environment not directly, and not in their entirety, but rather by way of specialized sense organs. One of them is the eye. Environmental influences give to it the corresponding information and that information goes to the central nervous system. Whereas sensory impression is given by its modality sight, hearing, touch, taste and smell - and quality - for example, red, green, blue - its intensity is given by its quantity. The quantity of a sensory impression corresponds to the strength of the stimulus. The general nature of the response of a receptor to stimuli of increasing intensity appears in Fig. 1. The receptor potential becomes larger and the frequency of the triggered action potentials increases. Fig. 2 shows the relationship between stimulus intensity and the response elicited from a receptor. Such relations can be determined at various levels of the nervous system as well as for the subjective impressions and perceptions. The point of origin of the intensity/response curve is always an important characteristic; this is the smallest stimulus that just produces a response, the threshold stimulus $S_{0}$. As a consequence of these facts,

\subsection{Ocular dominance columns}

The most detailed map of the retina is found in area V1 of the mammalian visual cortex. This detail goes close with a rich functional architecture, uncovered by Hubel and Wiesel in their physiological studies ${ }^{1}$.

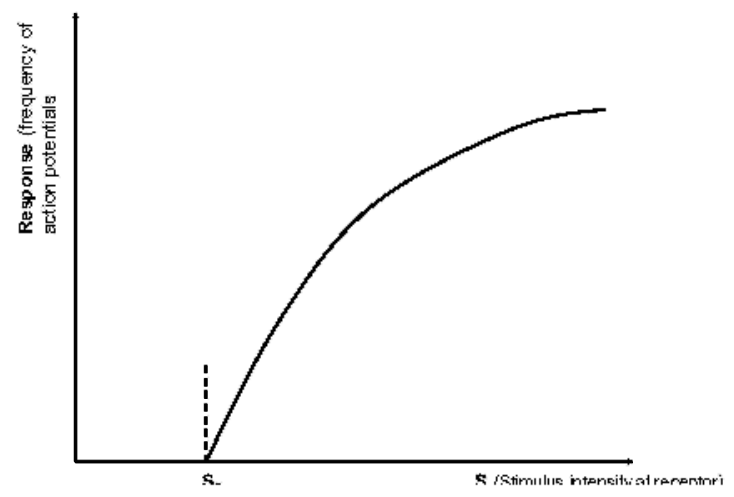

Fig. 2.- Dependence of the frequency of action potentials upon stimulus strength
They proposed that V1 was made up of a set of orientation columns and a set of ocular dominance columns. A way to clarify these ideas is to see how cells encountered in a penetration made perpendicular to the cortical surface of V1 respond.

Some cortical visual neurons have receptive fields with "on" or "off' zones arranged in parallel. Diffuse illumination of the entire receptive field causes little change in the spontaneous activity of them. But if a "bar" of light in the "correct" orientation and position is projected into the receptive field, there is strong activation. These fields are called "simple" receptive fields, because it is easy to establish their functional organization by projecting small spots of light onto different parts of the receptive field.

Other type of neurons with "complex" receptive fields covers higher order functions. The whole structure and function 
of the neuron primary visual cortex architecture is somehow much more complex. Their nerve cells are arranged in six

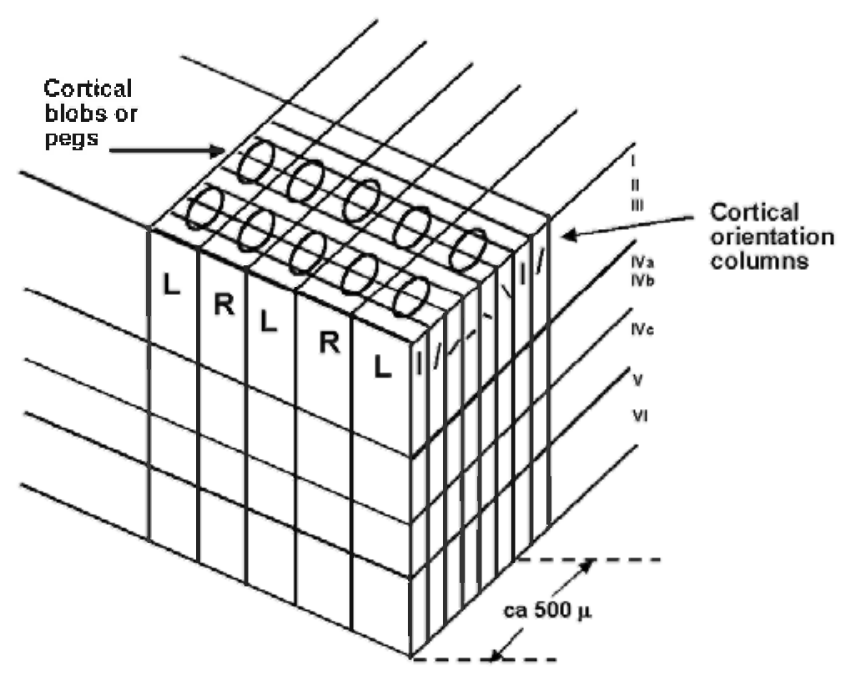

Fig. 3.- Structure of a part of the mammalian visual cortex. distinct cytoarchitectonic layers parallel to the cortical surface. These nerve cells are organized not only within layers parallel to the surface but also in "columns" perpendicular to it. When the neurons laying at these columns are tested with contours oriented in various directions one finds a progressive change in optimal orientation across adjacent columns. In other words, the cells in a particular column share the same preferred orientation. This orientation changes in a systematic way as one moves across the cortical surface, such that after half a millimeter or so we are back to the first orientation. Thus the visual cortex is traversed by a series of bands, within each of which every possible orientation is represented. This fact may be demonstrated anatomically. A highly stylized representation of a slab of visual cortex, showing its organization into columns, is shown in Fig. 3. It is necessary to add that vision corresponding to right and left eyes are at alternate columns. It is necessary to travel a minimum distance of about $0.8 \mathrm{~mm}$ for the cells to respond to the right eye plus the left eye and then start the sequence again, a distance comprising the orientation hypercolumn. Equally, it is necessary to travel about $1 \mathrm{~mm}$ for all 18 orientations to be covered, a distance comprising the orientation hypercolumn. Each small part of the field of view is screened for one eye and then for the other eye, and each small part is simultaneously screened for different orientations, the entire process being repeated again in the adjacent millimeter for an adjacent small part of the field of view. Colors are detected, in a similar way, at specific places, named cortical blobs or pegs. This structure is sometimes called the "ice-cube" model. A simplified model appears in Fig. 4.

The extraordinary regularity and precision of the retinal map in V1 may have misled to some people into thinking that it must be the retina that is mapped in all visual areas. But the results from studies of the prestriate cortex suggest that it may need a new concept of what a map in the cortex means. Some people are inclined to think of these maps as static maps of the retina. But it may be useful to think of them as dynamic maps and even constructional maps.

The relation between the external image and image the living body "sees" may have some to do with these aspects of the visual cortex.

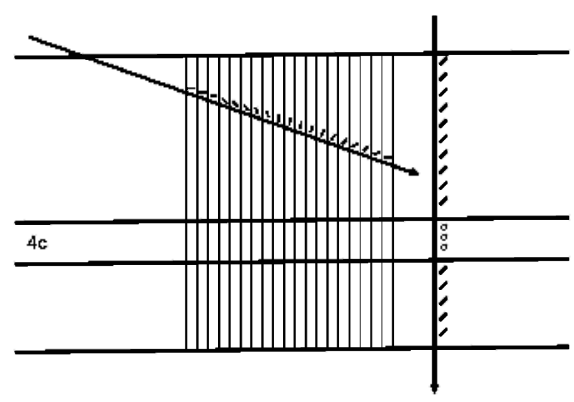

Fig. 4.- Simplified vision of the "ice-cube" model.
Without going into more details, we consider that these facts are related in a direct way with the interpretation human beings perform when some subject impinges onto their visual systems. Each direction, in this case, activates a particular column and a nervous current will be processed. If two excited columns are excited and they are close to each other, the total current towards higher sensory systems may be different than just a simple addition of two currents. Some influence between them must be present and hence, a different impression has to be obtained. These facts will be taken into account in the model we propose in the next paragraph. More details about these physiological aspects of the vertebrate visual system may be seen in any physiology textbook. See, for example ${ }^{2-4}$.

\subsection{Synthesis of main factors to be considered}

Although some other factors should be considered, in order to remember some of the vertebrate nervous system characteristics, 
we present now some of the main factors to considerer in present work. These points, in a very synthesized form are the following:

a. Different aspects of the visual scene, as seen by living beings, are perceived in a parallel way.

b. Each portion of the visual scene goes through the different layers of the retina by different paths.

c. Information about the relation between the information obtained from a particular area and information from adjacent areas is transferred to following layers, by a particular frequency coding in adjacent neuronal paths - this aspect has not been considered in previous paragraphs.

d. Information about the intensity of each one of the scene details are transferred from the third retina layer to following levels after a conversion from intensity level to frequency. Lower intensities correspond with lower frequencies.

e. Information about different line orientations is transferred to selected areas at the visual cortex. These areas become excited by this information and living beings get a stimulus concerning that orientation in the visual scene.

f. Each particular orientation, or each particular shape, goes to a precise area in the V1 area of the visual cortex.

g. Information is always transferred in a parallel way.

h. Visual cortex, at area V1, gets a "virtual image" of the real image appearing in the scene as "seen" by the living beings.

i. No "biological" software appears at the visual cortex. Any type of information is processed by hardware interconnections among different neurons.

j. Any biological information processing is performed by non linear effects.

k. Although different types of information processing appear in the retina and visual cortex this processing is the result of interchange of information among neurons in the same level. No feedback processes appears in the neural network.

1. The number of levels needed to go from receptor neurons in the retina to V1 layer in the cortex is lower than 15 .

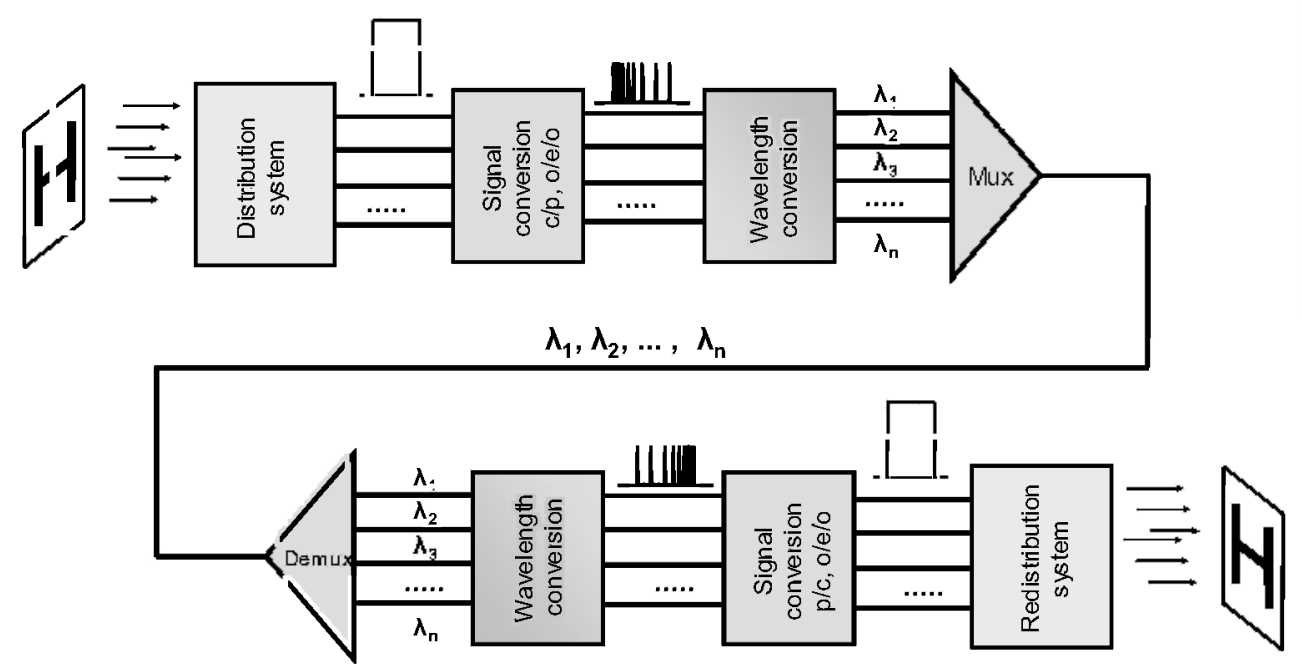

Fig. 5.- General diagram of the WDM image transmission system. 
These facts will give the main ideas to be implemented in our system.

\section{PROPOSAL FOR AN ARTIFITIAL SYSTEM EMULATING THE MAMMALIAM VISUAL CORTEX}

As it was pointed out before, the main purpose of this paper is to report a proposal for an image transmission system emulating the way mammalian visual cortex works. The main advantage of such a system is the possibility to transmit and recognize images without the need of any specific software or algorithm. A system as the one will be presented here will have the advantage of processing images in a parallel way and, at the same time, to reduce the number of steps, needed to perform such a task, to a very low value. The operations to be carried out will be the conventional functions had in any mammalian sensory system, as seen in previous paragraphs. Moreover, the levels of the image representation to be reported will be just the first ones. We restrict our present model to the path between the first levels of the retina to the first levels of the visual cortex. This means that our image transmission corresponds to the interval between the initial entrance onto the photoreceptors and this image going into the ocular columns at the cortex. Details to be analyzed are the simplest. However, a similar method to the one reported here could be easily implemented to recognize more complex shapes or structures.

The general structure to be employed is the one shown in Fig. 5. The bases are the 12 points indicated at the end of previous paragraph. The main objective is to transfer a particular image - a " $\mathbf{H}$ " in this figure - to a receptor. Distance between a transmitter and a receiver is not a limitation for our system. The structure is composed by different blocks, each one with a different function. Details about internal structure will be given afterwards. At this moment the principal aspect to be considered is the way we have implemented the parallel way of information transmission. The adopted solution has been by identification of every one of the parallel paths by a different wavelength. In this way, different neuronal paths may go through the same physical path and each one of the signals may be processed in an individual basis. This WDM method allows us to handle as many different signals as optical frequencies are available. As it will be indicated later, each one of these wavelength corresponds to each one of the different line orientations or shapes to be analyzed.

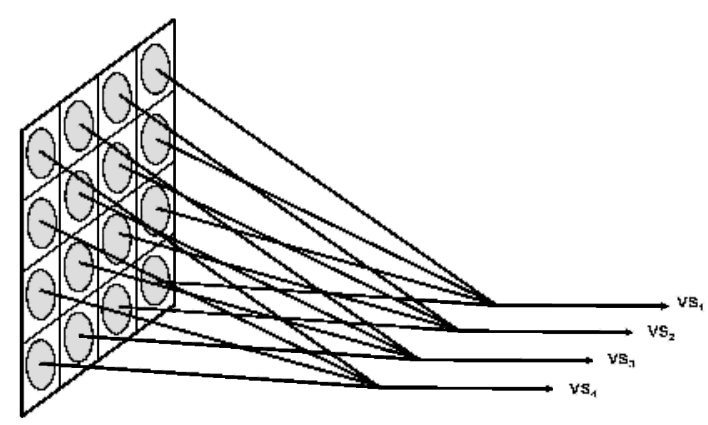

Fig. 6.- Outputs from the sensing area corresnondino to vertical orientations

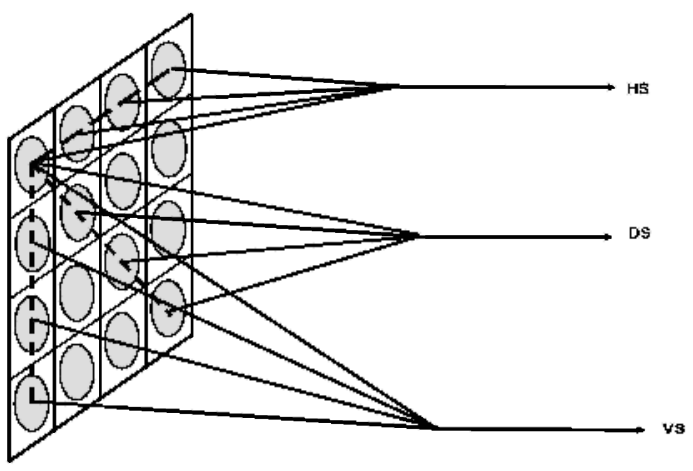

Fig. 7.- Outputs from one sensing unit corresponding to three different orientations 


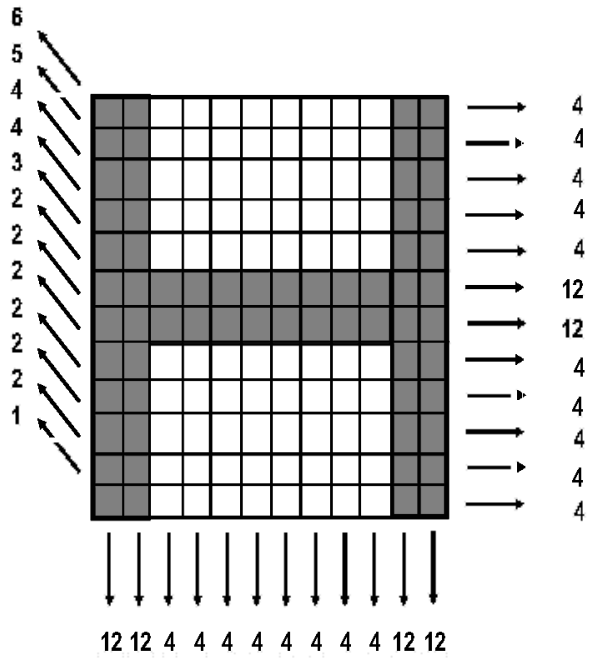

Fig. 8.- Intensity levels from signals obtained from an "H".

Other units at the center of the sensing area would have four different outputs: two corresponding to vertical and horizontal directions and two more for $+45^{\circ}$ and $-45^{\circ}$ orientations with respect to the vertical line. If different lines or shapes should be detected, different outputs connections between units should have been adopted. Fig. 6 shows the way similar sensing units - vertical lines in the present case - are connected.

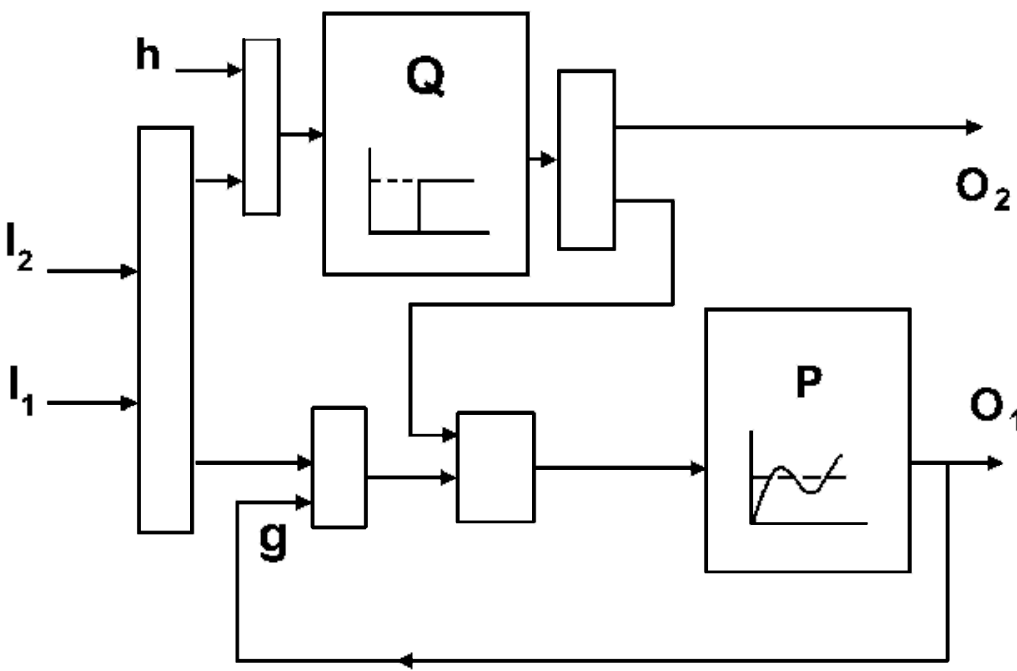

Fig.9.- Optically Programmable Logic Cell main blocks
Equivalent connections are present for other lines or shapes. From

above considerations, any signal coming to a sensing unit is the origin of a certain number of data channels. Each one of them corresponds to information about the received input. This information may or not be of interest by itself. It is the relation between it and signals received by other sensing units around that is of interest. In any artificial vision system, similar information obtained from a receiver is a part of the final signal. In our case, this signal will be important if belongs to a welldefined line or shape. But it is not the same in any other case. This is the difference between both types of sensing systems, artificial and biological.

In order to clarify above 
concepts, fig. 8 shows the signals obtained from a certain letter, in this case an " $\mathrm{H}$ ". We have adopted a sensing area composed by a $12 \times 12$ elements and we have supposed that full signal goes to a sensing unit. If this is not the case, it does not receive any signal at all. Intermediate cases require a further analysis and will be considered in another occasion. Possible output wiring corresponds here to 12 vertical lines and 12 horizontal lines. We have added, as well, 12 possible more orientations, at $-45^{\circ}$. In the same way, another set of twelve $+45^{\circ}$ orientations should be present at the right side of the rectangle as well as other similar orientations along other sides. Numbers indicate the level of the final signal at each one of the possible outputs. A full line corresponds to a level - in our present example - of 12 arbitrary units. Other numbers determine that the full line is not covered by the image. For instance, number 4 indicates that just four sensing units have received signals. Similar reasoning would be applied to directions forming a certain angle with respect to vertical or horizontal lines.

The straightforward case is when just full lines are considered. In this case, a thresholding element should eliminate signals no reaching the maximum value. Only information concerning this type of lines, with signal reaching full levels, would be transmitted. Other cases will be considered further.

After the signals have overcome the distribution system they get the "Signal conversion system". Signals arriving there have a level corresponding to the quantity of light impinging to each one of the chosen directions or lines. They are, as we have shown, the result of the light reaching corresponding sensing units. Hence, information corresponds to multilevel signals. These signals,

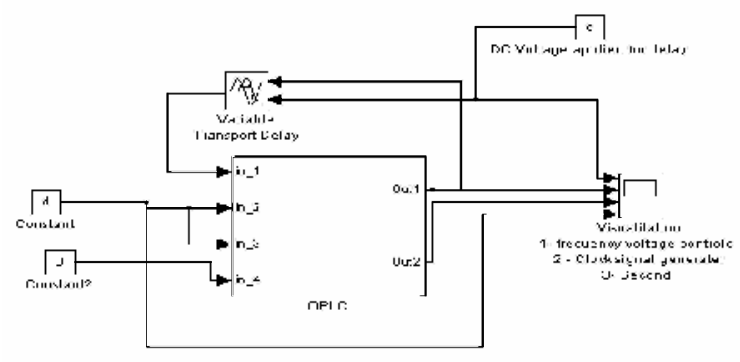

Fig. 10.- Basic configuration of the computer simulated voltage/frequency converter. according to the principles indicated previously, have to be converted to frequency dependent signals. Different intensity levels will correspond to different frequency signals with the same amplitude. Hence, the information will be transmitted now as burst of pulses with equal amplitude but different period. The way to perform this change in signal characteristics is by the use of Optical Programmable Logic Cells, OPLC, previously employed by us in optical computing and as optical digital chaos generators (see, for example ${ }^{5-7}$ ). A scheme of this type of structure is shown in Fig. 9 . As it can be seen, it is composed by two devices with a non-linear behaviour, $\mathrm{P}$ and $\mathrm{Q}$, the outputs of each one of them correspond to the two final outputs, $\mathrm{O}_{1}$ and $\mathrm{O}_{2}$, of the cell. Four are the possible inputs to the circuit. Two of them are for input data, $\mathrm{I}_{1}$ and $I_{2}$, and the other two, $\boldsymbol{g}$ and $\boldsymbol{h}$, for control signals. The method to obtain periodic outputs is - as it may be seen in Fig. 9 - to introduce a feedback between an output gate of the OPLC and one of the possible inputs. In our present case we have taken the output corresponding to the $\mathrm{P}$ device. This feedback introduces a time delay. This
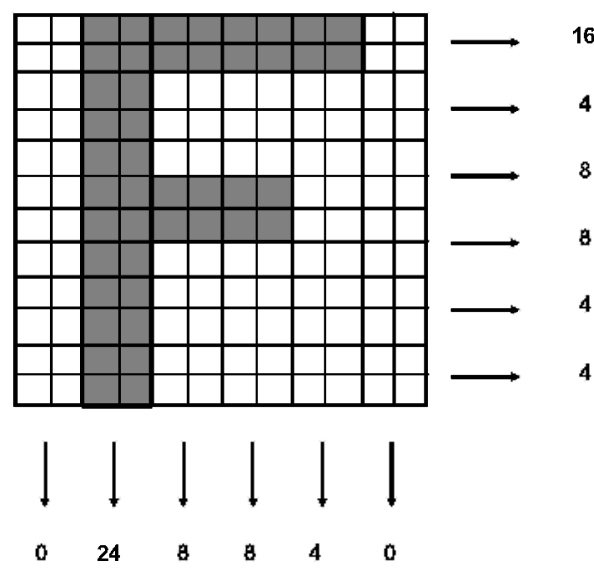

Fig. 11.- Intensity levels frōm signals obtained from an " $F$ ". time delay determines the frequency of the oscillation. Moreover, it is necessary to introduce an input signal. In the present case, this signal is a continuous obtained from a step function. It is important to recall that the oscillation frequency is a function of the introduced time delay. This time delay has been designed in such a way that the signal need to set up its configuration, determines the delay and, as a consequence, the frequency. This configuration works as a VCO (Voltage Controlled Oscillator). The voltage, in our present situation, is given by the signals coming from the Distribution system. The implemented system, as it was computer simulated, appears in Fig. 10 where the main blocks are present. The corresponding DC Voltage applied for delay is a faction of the signal obtained from a particular orientation line.

Next block in Fig. 5 is "Wavelength conversion". This step offers several possibilities. 
As a matter of fact, it may be located previously to the intensity/frequency conversion. The light impinging to sensing units, coming from the object, is common light. Hence, it is necessary to convert it to monochromatic light. This might
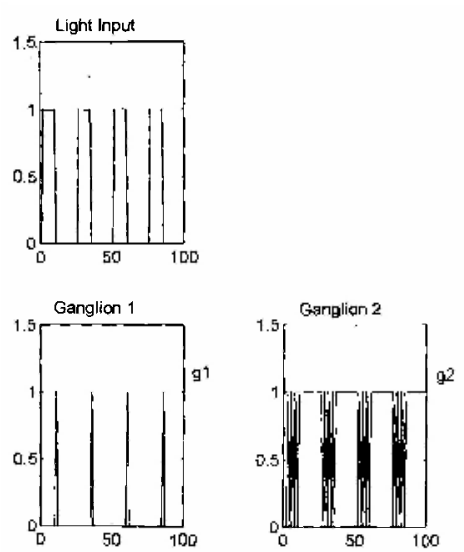

Fig. 12.-
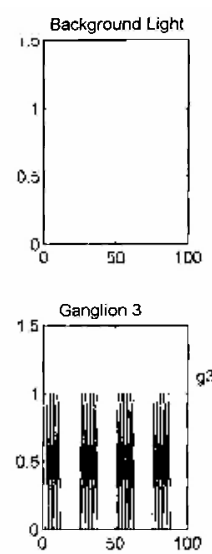

be done after of previously to the intensity/frequency conversion. Any solution is possible and each one has advantages and disadvantages. We have adopted the solution indicated in Fig. 5. The main objective is to transmit each one of the directions in the initial image with a different wavelength. This solution allows the parallel transmission appearing in biological systems. The indicated multiplexer collects different signals from different orientations and are transmitted along the same optical channel. Hence, a WDM transmission offers a way to send information in an equivalent form to the parallel transmission in living beings.

Reception is equivalent to the emitter side with a symmetrical configuration. Similar steps appear now in inverse order. Demultiplexing may be done by any conventional method. Different wavelengths split and change from signals with a certain frequency to signals with different intensities. The more important part in reception now is routing these different optical frequencies to the corresponding address. Wavelengths coming from horizontal orientations have to be applied to the same addresses they had in the initial image. The correspondence between initial and final wiring is the more important part of the system. Orientations or lines excited at the initial stage have to correspond with equivalent orientations or lines at the

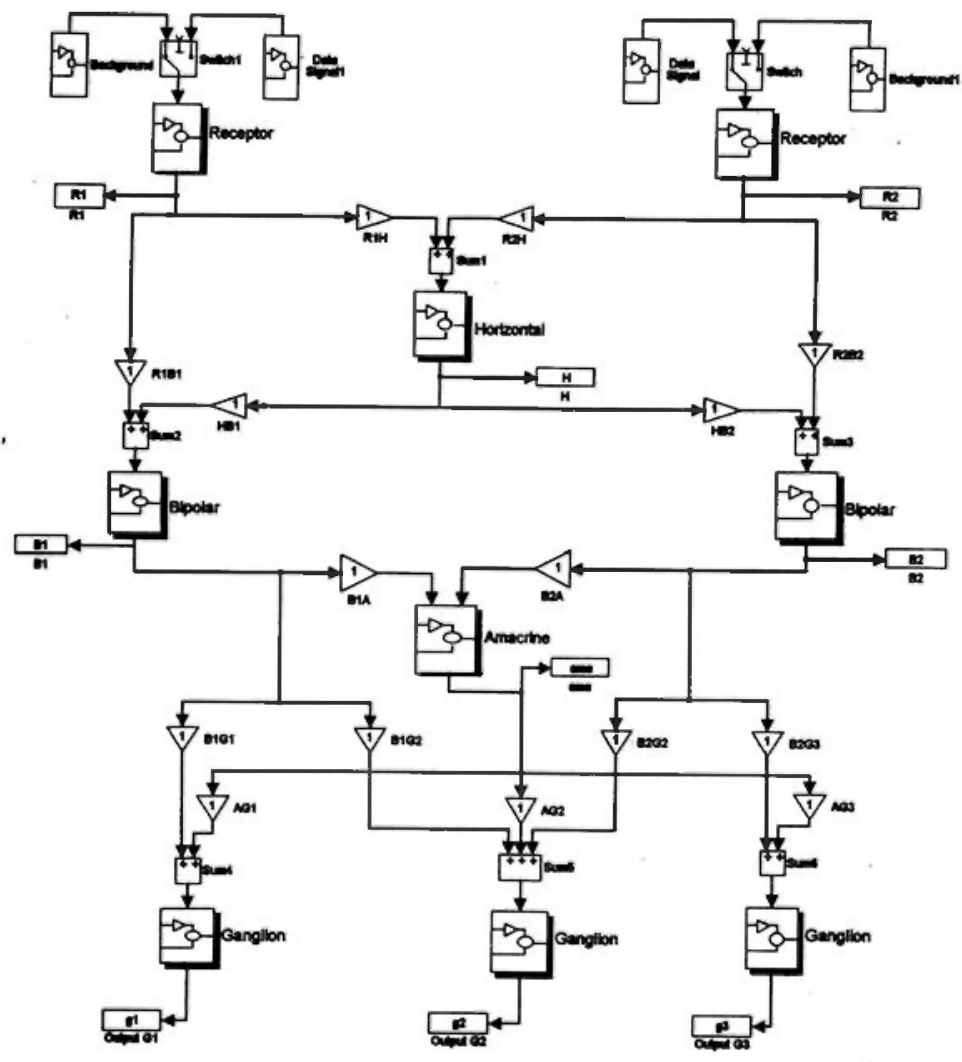

Fig. 5-Computer simulation of the retina model. final stage. Because the signal before the redistribution system has a level corresponding to full covered lines, a simple intensity divider distributes incoming signal to the different reception units. Hence the transmission of image has been achieved.

\section{FURTHER MODIFICATIONS OF THE PROPOSED SYSTEM}

The reported system corresponds with the simplest configuration to transmit images by a WDM system. Above indicated method is able to carry information about simple lines or directions when each one of the sensing units is either fully illuminated or without any signal at all. Variations with respect to this situation would affect to emitter and receiver. However, transmission is - as it was described before - a common part and any possible modification implemented to improve the system characteristics would maintain it without changes. Hence, we will indicate now some minor modifications to previous system concerning mainly emitter and receiver.

A sensing area, fully composed of sensing units, may receive some image covering just a part of its surface and partly covering some of its sensing units. This situation corresponds with example shown 
in Fig. 11. Matrix has been reduced to $6 \times 6$ and each one its sensing units are partly covered with signal. In this occasion, intensity levels obtained from every one of the corresponding directions are a function of the covered surface. We have assumed that a sensing unit fully covered would give intensity 4 . The analysis of this situation is much more complex than before because it is not possible to obtain direct information about lines by simple "yes" or "not". But, once more, solutions adopted by living bodies give indications about how to proceed. This situation corresponds with the very well known synaptic connections in sensing organs as, for example, retinas and visual cortex. It corresponds to several possible configurations for cells known as "On-center" and "Off-center". The response of these types of cells depends on the light impinging on the cells around them. A detail of this behavior may be seen at the literature ${ }^{8-10}$. The main factor is that the firing rate of a certain cell, as a response to a spot of light, depends on the light impinging on the cells around. Hence, it possible to know whether a receiving cell gets light as well as the cells around it or if this cell receives light but cells around remain in the darkness. This fact provides information to be processed in later steps.

Another point concerns scenes changing in time. Up to now every image has been considered with static conditions. But images are changing and sensing elements must get information bout that. Two facts may be considered. First one is how many elements have changed in a certain scene. If just a part of the image has changed, no new information has to be transmitted concerning areas where data are similar to previous ones. Second fact relates synchronizing information transmitted through different channels. This synchronism is due to the need of coordinating changes in different unit areas of the whole image.

Once more, retina gives some clues to solve above indicated problems. If light impinges onto two adjacent photoreceptors, and attention in given to ganglion cells carrying this information the visual cortex, depending on the light intensity ratio between these two photoreceptors, different outputs appear at the ganglion dendrites. An example appears in Fig. 11 where one receptor gets light and the other remains in darkness. As it may be seen, three different outputs appear. This situation has been analyzed by Dowling ${ }^{8}$ and has been implemented by us ${ }^{11}$. Fig. 12 shows the computer simulation of this structure. The whole architecture is based on the same unit appearing in Fig. 9. Every one of the blocks in Fig. 12 has the same configuration. A very important fact about its behavior is that just by simple changes in the internal connections, either in the elemental blocks or between or between them a large variety of responses may be obtained. These outputs give the possibility to add the needed information. A whole system, with these facts together, will be reported by us.

\section{CONCLUSIONS}

A new method to transfer images from one emitter to a receiver has been reported in this paper. Its main characteristic is the adopted model: the mammalian visual system. It offers the possibility, due to this fact, to transmit parallel information about simple images by using WDM technologies. Moreover, simple lines are directly transferred and a simple way to detect changes in the initial scene is possible.

The reported model, although in a very initial form, offers several advantages with respect to conventional methods. The fist one is the possibility to transmit directly any shape or line without any need to select parts of it and recollect them in later steps. This action is just done by a particular "hardware" arrangement after the sensing unit. Information about each chosen direction or line is transmitted in a simple way. Moreover, each one of these chosen details is transmitted by a different wavelength. This situation is equivalent to the parallel transmission had in living bodies. A similar arrangement in the receptor recovers the information sent by the emitter.

As it has been indicated, the reported model is in a very elemental form. We have adopted its more simplified version of it in order to present the fundamental lines. Just the basic ideas have been reported as well as some ways to implement them in a real structure. Previous works by us, related with the mammalian retina and visual cortex, have been adopted as main parts of this structure. We have not gone into details of their characteristics because have been published previously in several places. But indications about its way to be implemented and the problems they solve with respect to conventional methods have been indicated.

\section{ACKNOWLEDGMENTS}

This work was partly supported by CICYT "Comisión Interministerial de Ciencia y Tecnología”, grant TIC991131 and CAM "Comunidad Autónoma de Madrid", grant 07T/0037/2000. 


\section{REFERENCES}

1. D.H. Hubel and T.N. Wiesel, "The Ferrier Lecture: Functional architecture of macaque monkey visual cortex", Proc. R. Soc. London. B 198, 1-59 (1977).

2. G.M. Shepherd, "The Synaptic Organization of the Brain". Oxford. 1990.

3. "Neuro-vision Systems. Principles and Applications". Eds.: Madam M. Gupta and George K. Knopf. IEEE Press. New York. 1993.

4. J.G. Nicholls, A. Robert M. and B.G. Wallace, "From Neuron to Brain", Sinauer Associates, Inc. Sunderland, MA. 1992.

5. A. González-Marcos \& J.A. Martín-Pereda, "Digital Chaos Synchronization In Optical Networks". In "OPTICAL NETWORK DESIGN AND MODELLING II". Eds.: G. de Marchis y R.Sabella. Kluwer Academic Publishers, pp. 175-186. 1999.

6. A. González-Marcos \& J.A. Martín-Pereda, "Analysis of irregular behaviour on an optical computing logic cell". Optics \& Laser Technology, 32, 45557 - 466 (2000)

7. A. González-Marcos and J.A. Martín-Pereda, "Method to analyze the influence of hysteresis in optical arithmetic units". Optical Engineering, 40, 2371-2385 (2001)

8. J. E. Dowling, "The Retina: An Approachable Part of the Brain". The Belknap Press of Harvard University Press. Cambridge, MA (1987).

9. G.M. Shepherd, "Neurobiology", Oxford University Press. New York (1994)

10. "Neuro-vision Systems. Principles and Applications". Eds.: Madam M. Gupta and George K. Knopf. IEEE Press. New York (1993).

11. A. González-Marcos and J.A. Martín-Pereda, "A New Approach to a Model of the Mammalian Retina with Optically programmable Logic Cells". $1^{\text {st }}$. International IEEE EMBS Conference on Neural Engineering. Capri (Italia). 20-22 de marzo, 2003. ISBN: 0-7803-7819-9 